\title{
BCR-JAK2 Fusion in Hematologic Malignancies
}

National Cancer Institute

\section{Source}

National Cancer Institute. BCR-JAK2 Fusion in Hematologic Malignancies. NCI Thesaurus. Code C157387.

An extremely rare finding in hematologic malignancies indicating an association with BCR-JAK2 fusion gene that results from t(9;22)(p24;q11.2). Most cases exhibit chronic myelogenous leukemia-type phenotype. 